\title{
Designing highly efficient solvents for the Knoevenagel condensation: two novel dicationic dimethyl phosphate ionic liquids
}

\author{
Elina Priede,* Sindija Brica, Niklavs Udris, and Andris Zicmanis \\ University of Latvia, Faculty of Chemistry, 19, Rainis Blvd., Riga, LV1586, Latvia \\ E-mail:priede_elina@inbox.lv
}

DOI: $\underline{\text { http://dx.doi.org/10.3998/ark.5550190.p009.355 }}$

\begin{abstract}
Two novel dicationic dimethyl phosphate ionic liquids have been designed as highly efficient solvents for the Knoevenagel condensation between ethyl cyanoacetate and aldehyde with a decreased reactivity - 4-(dimethylamino)benzaldehyde. A simple synthetic strategy has been demonstrated for obtaining the dicationic dimethyl phosphate ionic liquid bearing both aromatic imidazolium and aliphatic ammonium moieties.
\end{abstract}

Keywords: Dicationic ionic liquids, dimethyl phosphates, anion metathesis, Knoevenagel condensation

\section{Introduction}

The Knoevenagel condensation is a significant $\mathrm{C}-\mathrm{C}$ bond forming reaction that provides a wide range of key intermediates for the synthesis of pharmaceuticals, polymers, cosmetics and perfumes. ${ }^{1}$ This aspect has aroused a particular interest in developing new and advantageous synthetic approaches towards this reaction. Different molecular solvents, ${ }^{2}$ catalysts (organic bases, ${ }^{3,4}$ heterogeneous catalysts, ${ }^{5}$ Lewis acids ${ }^{6}$ ) and reaction conditions (microwave ${ }^{7}$ or ultrasound $^{8}$ irradiation) have been applied to ensure sufficiently high yields of the target products. Nevertheless, the utilization of toxic solvents or catalysts and prolonged reaction times are inconsistent with the principles of green and sustainable chemistry ${ }^{9}$ and still have to be overcome. Ionic liquids (ILs) - structurally divergent compounds with beneficial and finetunable properties $^{10,11}$ - have recently been presented as alternative and efficient solvents or catalysts $^{1,12,13}$ for environmentally benign Knoevenagel condensations.

As a distinct group, dicationic ionic liquids (DILs) frequently possess enhanced chemical and physical properties, ${ }^{14,15}$ as well as decreased toxicity ${ }^{16}$ in comparison to the respective monocationic ILs. Several DILs have been successfully applied in the Knoevenagel condensation. Mane and co-workers have performed the DIL mediated synthesis of 5-arylidine- 
2,4-thiazolidinediones. ${ }^{17}$ Luo and co-workers have exploited PEG bridged tertiary amine functionalized DIL for the condensation between benzaldehyde and ethyl cyanoacetate. ${ }^{18}$ Godajdar and co-workers have presented the synthesis of $1 H$-indazolo[2,1-b]phthalazinetrione, catalyzed by a magnetic DIL. ${ }^{19}$ Our group has recently developed a method for obtaining DILs with a dimethyl phosphate counterion. ${ }^{20}$ Several examples of condensation reactions, promoted by imidazolium-based dimethyl phosphates, ${ }^{21,22}$ encouraged us to continue our earlier studies and to demonstrate the possibility of designing some dicationic dimethyl phosphate ILs as efficient solvents for the Knoevenagel condensation.

\section{Results and Discussion}

Herein, we report the synthesis of two novel DILs - 1,4-bis(3-methylimidazolium-1-yl)butane bis(dimethyl phosphate) (4) and 1-[2-(diethylmethylammonium)ethyl]-3-methylimidazolium bis(dimethyl phosphate) (5) (Figure 1), as well as their application in the Knoevenagel condensation between 4-(dimethylamino)benzaldehyde (6) and ethyl cyanoacetate (7) (Scheme 1). To develop a highly efficient solvent, an aldehyde bearing an electron-donating substituent and therefore exhibiting a lower reactivity ${ }^{23}$ was selected as the electrophilic reagent for this model reaction and no additional catalyst was used. The impact of ILs was assessed by the conversion of 4-(dimethylamino)benzaldehyde (6) and the results obtained via gas chromatography were in a full agreement with the respective isolated yields of the product $\mathbf{8}$.

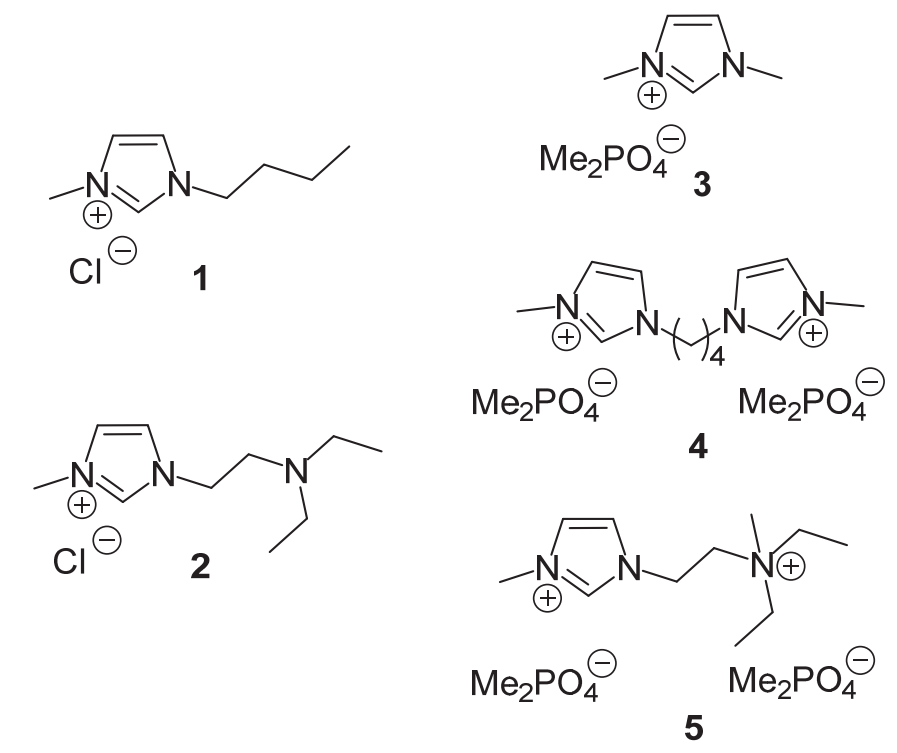

Figure 1. The imidazolium-based ILs 1-5 exploited as solvents for the Knoevenagel condensation outlined in Scheme 1. 


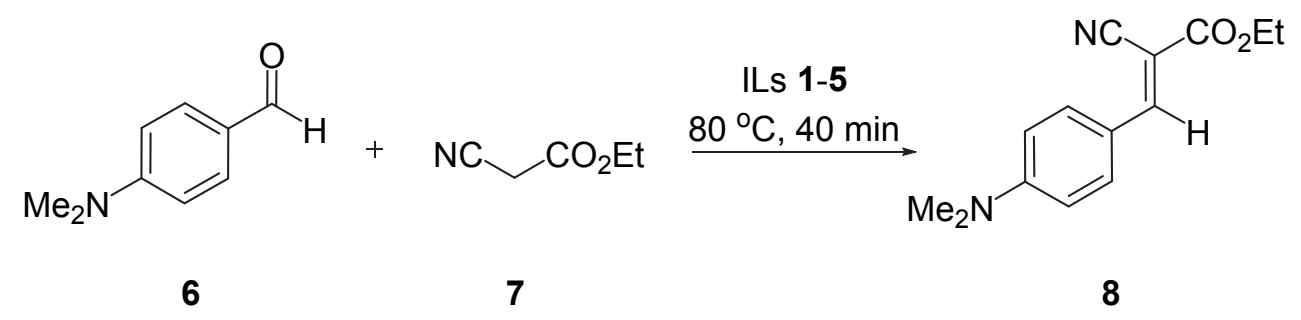

Scheme 1. The Knoevenagel condensation between 4-(dimethylamino)benzaldehyde (6) and ethyl cyanoacetate (7) in the medium of ILs 1-5.

We started our investigation using 1-butyl-3-methylimidazolium chloride (1), and the conversion of aldehyde 6 after $60 \mathrm{~min}$ at $80{ }^{\circ} \mathrm{C}$ in this solvent had reached only $17 \%$ (Figure 2). Due to the fact that introduction of a basic functionality into the cation of IL may have a significant impact on some base catalyzed reactions, ${ }^{24,25}$ IL 2, having [2-(diethylamino)ethyl]group in the imidazolium moiety, was evaluated as a potentially more appropriate solvent. Therefore, a simple and mild approach for the synthesis of this salt has been demonstrated (Scheme 2) in the frame of this work. The IL 2 could be easily obtained by the deprotonation of 1-[2-(diethylamino)ethyl]-3-methylimidazolium chloride hydrochloride (9) with $\mathrm{NaOMe}$ in $\mathrm{MeOH}$ at room temperature. However, a slight excess (1.2 equiv) of the dicationic salt 9 was crucial for obtaining a colorless IL 2, indicating that any residual $\mathrm{NaOMe}$ may be responsible for the coloration of the target product. This may be caused by the acidic nature of the imidazolium ring $\mathrm{C}^{2}$ proton that may lead to the formation of highly reactive $N$-heterocyclic carbene impurities. ${ }^{26,27}$

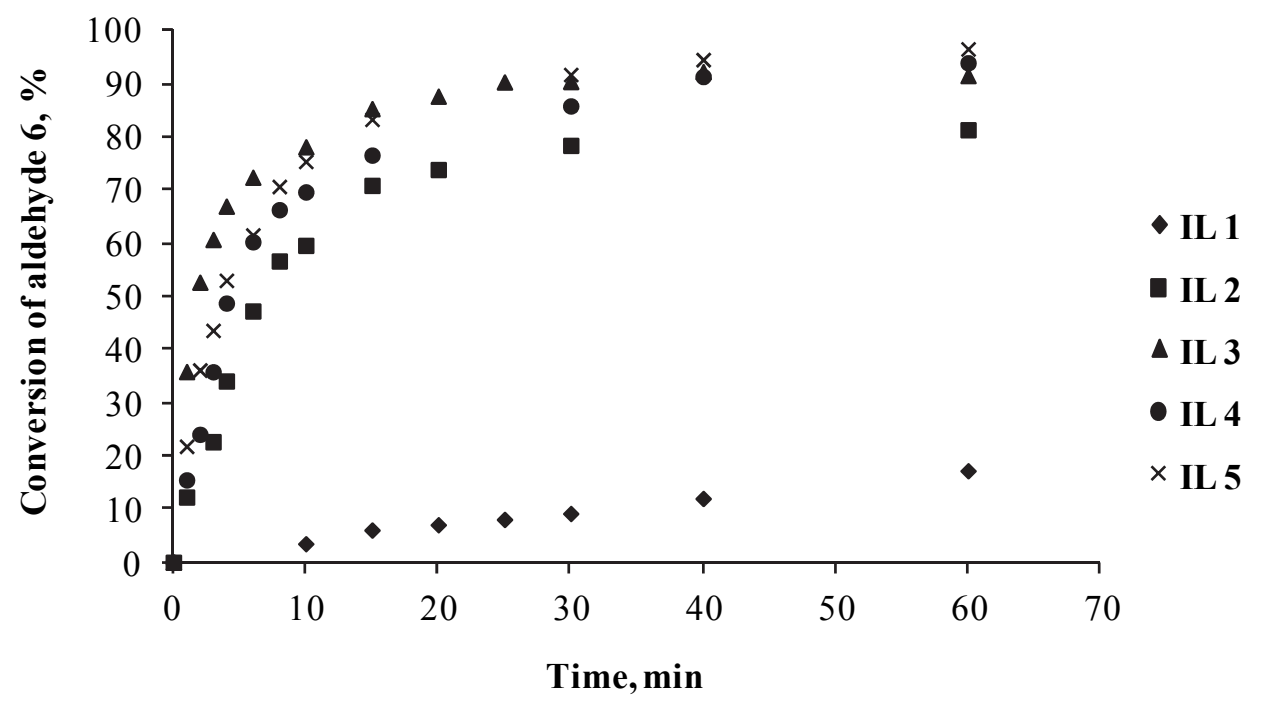

Figure 2. The conversion of 4-(dimethylamino)benzaldehyde (6), performing the Knoevenagel condensation in ILs $1-5$ at $80^{\circ} \mathrm{C}$. 

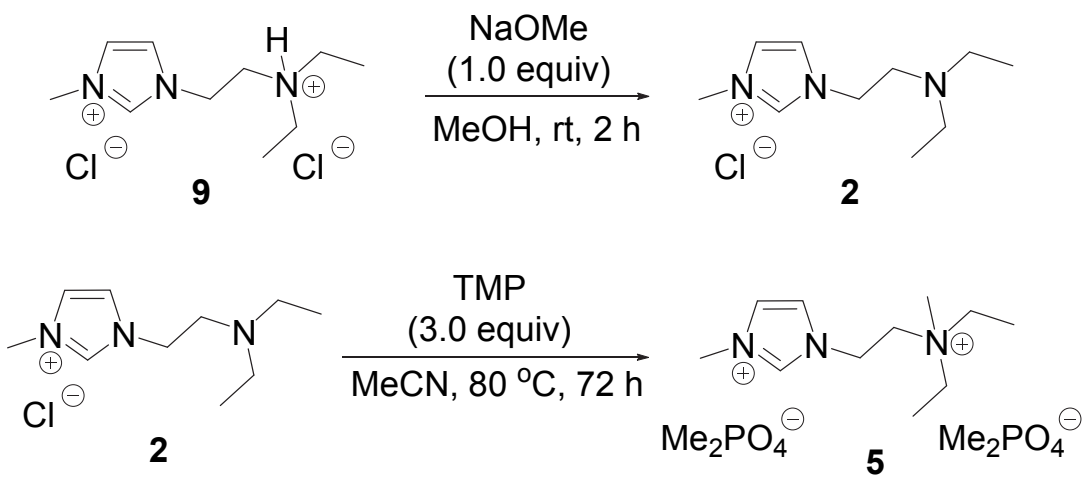

Scheme 2. The synthesis of 1-[2-(diethylmethylammonium)ethyl]-3-methylimidazolium bis(dimethyl phosphate) (5).

Hence, the application of 1-[2-(diethylamino)ethyl]-3-methylimidazolium chloride (2) allowed improving the conversion $(81 \%$ after $60 \mathrm{~min})$ of the aldehyde $\mathbf{6}$. Nevertheless, 1,3dimethylimidazolium dimethyl phosphate $(\mathbf{3})^{22}$ as a solvent provided even higher conversion ( $92 \%$ after $60 \mathrm{~min}$ ) of the starting material. These aspects clearly showed that structural tuning, with correct selection of the cation and anion of IL, may lead to an efficient solvent for this reaction. Thereby we were encouraged to design two novel DILs with dimethyl phosphate anion. 1,4-Bis-(3-methylimidazolium-1-yl)butane bis(dimethyl phosphate) (4) was synthesized according to the procedure reported by our group ${ }^{20}$ and provided an excellent conversion $(94 \%$ after $60 \mathrm{~min}$ ) of the aldehyde 6. Furthermore, a synthetic strategy for obtaining 1-[2(diethylmethylammonium)ethyl]-3-methylimidazolium bis(dimethyl phosphate) (5) was developed. A simple procedure, performing a simultaneous metathesis of the chloride ion and alkylation of the tertiary amine in IL 2 with trimethyl phosphate (TMP) (Scheme 2), provided IL 5 in quantitative yield (99\%). Despite the lack of a basic $\operatorname{site}^{28}$ in the cation after quaternization, the DIL 5 consequently provided excellent conversion (97\% after $60 \mathrm{~min}$ ) of the aldehyde 6. Although the conversion of 4-(dimethylamino)benzaldehyde (6) in the medium of ILs 3-5 is similar, our attempts to clarify the suitability of dicationic dimethyl phosphate ILs for the Knoevenagel condensation have resulted in the development of two previously unreported compounds. Since DILs usually have enhanced chemical and physical properties ${ }^{14,15}$ and decreased toxicity ${ }^{16}$ in comparison to monocationic ILs, these new DILs might be a subject of interest in the context of environmentally friendly organic synthesis that benefit from the structural peculiarities of these ILs.

\section{Conclusions}

Two novel dicationic ionic liquids (DILs) - 1,4-bis-(3-methylimidazolium-1-yl)butane bis(dimethyl phosphate) (4) and 1-[2-(diethylmethylammonium)ethyl]-3-methylimidazolium 
bis(dimethyl phosphate) (5) - have been designed as highly efficient solvents for the Knoevenagel condensation between 4-(dimethylamino)benzaldehyde (6) and ethyl cyanoacetate (7). A simple synthetic strategy involving a simultaneous metathesis of the chloride ion and alkylation with trimethyl phosphate (TMP) has been developed for obtaining 5 that possesses both aromatic imidazolium and aliphatic ammonium moieties. Compared with monocationic ILs bearing a chloride anion, dicationic dimethyl phosphate ILs have provided excellent conversion of the aldehyde $\mathbf{6}$ without exploitation of any additional catalyst. In addition to the successful utilization of DIL 4, the divergent nature of DIL 5, having both aromatic imidazolium and aliphatic ammonium moieties and relatively basic anion, suggests that this new compound could also serve as a promising solvent for other reactions that benefit from these particular structural motifs within IL.

\section{Experimental Section}

General. All reagents were purchased from Sigma-Aldrich and used as received. Solvents (methanol, acetone, and toluene) were dried and purified according to standard procedures. ${ }^{29}$ The ${ }^{1} \mathrm{H}$ NMR (400 MHz) and ${ }^{13} \mathrm{C}$ NMR $(100 \mathrm{MHz})$ spectra (in $\mathrm{CDCl}_{3}$ or DMSO- $d_{6}$ ) were recorded on a Varian $400 \mathrm{MR}$ spectrometer ( $\delta$ in ppm, $J$ in Hz). The chemical shifts were reported relative to a residual solvent peak as an internal reference. The IR spectra were recorded on a PerkinElmer FT-IR/FIR Frontier instrument $\left(v_{\max }\right.$ in $\left.\mathrm{cm}^{-1}\right)$. The HRMS-ESI analyses were run on an Agilent 6230 TOF LC/MS mass spectrometer. The melting points were recorded on a Stuart SMP3 apparatus. The melting point of IL 5 was determined via DTA/TG as the onset temperature of the melting peak. The DTA/TG analysis was performed with a SII NanoTechnology Exstar6000 TG/DTA6300 instrument. Open aluminum pan was used. Sample $\left(\sim 10 \mathrm{mg}\right.$ ) was heated from 30 to $300{ }^{\circ} \mathrm{C}$ at a heating rate $10^{\circ} \mathrm{C} / \mathrm{min}$. The nitrogen flow rate was $300 \pm 20 \mathrm{~mL} / \mathrm{min}$. The conversion of 4-(dimethylamino)benzaldehyde (6) was calculated from the data obtained via gas chromatography. The analyses were performed on a YL6100 GC gas chromatograph equipped with a Restek Rtx ${ }^{\circledR}-1 \mathrm{MS}$ column $(30 \mathrm{~m} \times 0.25 \mathrm{~mm} \times 0.25 \mu \mathrm{m})$ and flame ionization detector (FID).

1-Butyl-3-methylimidazolium chloride (1) was obtained according to the known procedure. ${ }^{30}$ FTIR (ATR film), $v_{\max }\left(\mathrm{cm}^{-1}\right): 1561(\mathrm{C}=\mathrm{C}) .{ }^{1} \mathrm{H}$ NMR $\left(400 \mathrm{MHz}, \mathrm{DMSO}-d_{6}\right): \delta_{\mathrm{H}} 9.23(1 \mathrm{H}, \mathrm{s}$, NCㅌN), $7.79(1 \mathrm{H}, \mathrm{t}, \mathrm{NC} \underline{H C H N}, J 1.8 \mathrm{~Hz}), 7.72(1 \mathrm{H}, \mathrm{t}, \mathrm{NCHC} \underline{\mathrm{HN}}, J 1.7 \mathrm{~Hz}), 4.17$ (2H, t, $\left.\mathrm{NC}_{2}\left(\mathrm{CH}_{2}\right)_{2} \mathrm{CH}_{3}, J 7.2 \mathrm{~Hz}\right), 3.85\left(3 \mathrm{H}, \mathrm{s}, \mathrm{NC}_{3}\right), 1.76\left(2 \mathrm{H}\right.$, quint, $\left.\mathrm{NCH}_{2} \underline{\mathrm{CH}}_{2} \mathrm{CH}_{2} \mathrm{CH}_{3}, J 7.3 \mathrm{~Hz}\right)$, $1.26\left(2 \mathrm{H}\right.$, sext, $\left.\mathrm{N}\left(\mathrm{CH}_{2}\right)_{2} \mathrm{C}_{2} \mathrm{CH}_{3}, J 7.4 \mathrm{~Hz}\right), 0.90\left(3 \mathrm{H}, \mathrm{t}, \mathrm{N}\left(\mathrm{CH}_{2}\right)_{3} \mathrm{CH}_{3}, J 7.3 \mathrm{~Hz}\right) .{ }^{13} \mathrm{C} \mathrm{NMR}(100$

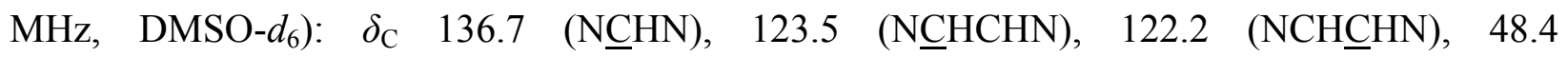
$\left(\mathrm{NCH}_{2}\left(\mathrm{CH}_{2}\right)_{2} \mathrm{CH}_{3}\right), 35.7\left(\mathrm{NCH}_{3}\right), 31.4\left(\mathrm{NCH}_{2} \underline{\mathrm{CH}}_{2} \mathrm{CH}_{2} \mathrm{CH}_{3}\right), 18.7\left(\mathrm{~N}\left(\mathrm{CH}_{2}\right)_{2} \underline{\mathrm{CH}}_{2} \mathrm{CH}_{3}\right), 13.3$ $\left(\mathrm{N}\left(\mathrm{CH}_{2}\right)_{3} \underline{\mathrm{CH}}_{3}\right)$. ESI-HRMS: $m / z[\mathrm{M}]^{+}$calcd for $\mathrm{C}_{8} \mathrm{H}_{15} \mathrm{~N}_{2}^{+}$: 139.1230. Found: 139.1216. 
1-[2-(Diethylamino)ethyl]-3-methylimidazolium chloride (2). Sodium (0.46 g, $20.0 \mathrm{mmol})$ was reacted with dry $\mathrm{MeOH}(20 \mathrm{~mL})$ under vigorous stirring and cooling in an ice bath. After the complete evolution of gas, a solution of 1-[2-(diethylamino)ethyl]-3-methylimidazolium chloride hydrochloride (9) (5.13 g, $20.2 \mathrm{mmol})$ in $\mathrm{MeOH}(30 \mathrm{~mL})$ was added dropwise and the reaction mixture was stirred at $\mathrm{rt}$ for $2 \mathrm{~h}$. The precipitated $\mathrm{NaCl}$ was filtered off on Celite ${ }^{\circledR} 512$ Medium and washed on the filter with $\mathrm{MeOH}(10 \mathrm{~mL})$. The combined filtrates were concentrated by rotary evaporation $\left(10 \mathrm{mbar}, 70{ }^{\circ} \mathrm{C}\right)$ and dry acetone $(30 \mathrm{~mL})$ was added to the obtained slurry. The mixture was repeatedly filtered through Celite ${ }^{\circledR} 512$ Medium and the precipitate was washed on the filter with acetone $(20 \mathrm{~mL})$. The combined solutions were filtered through a PTFE filter $(0.45 \mu \mathrm{m})$ and concentrated by rotary evaporation $\left(10 \mathrm{mbar}, 70{ }^{\circ} \mathrm{C}\right)$. The pure product was dried under vacuum $\left(0.6 \mathrm{mbar}, 75^{\circ} \mathrm{C}, 12 \mathrm{~h}\right)$.

IL 2. Colorless, glassy substance, yield 90\%, $3.92 \mathrm{~g}$; FTIR (ATR film), $v_{\max }\left(\mathrm{cm}^{-1}\right): 1670(\mathrm{C}=\mathrm{N})$, $1570(\mathrm{C}=\mathrm{C}) .{ }^{1} \mathrm{H}$ NMR $\left(400 \mathrm{MHz}, \mathrm{DMSO}-d_{6}\right): \delta_{\mathrm{H}} 9.18(1 \mathrm{H}, \mathrm{s}, \mathrm{NCHN}), 7.77(1 \mathrm{H}, \mathrm{t}, \mathrm{NC} \underline{H C H N}, J$ $1.7 \mathrm{~Hz}), 7.70(1 \mathrm{H}, \mathrm{t}, \mathrm{NCHC} \underline{\mathrm{HN}}, J 1.6 \mathrm{~Hz}), 4.20\left(2 \mathrm{H}, \mathrm{t}, \mathrm{NC}_{2} \mathrm{CH}_{2} \mathrm{NEt}_{2}, J 5.9 \mathrm{~Hz}\right), 3.87(3 \mathrm{H}, \mathrm{s}$, $\left.\mathrm{NCH}_{3}\right), 2.71\left(2 \mathrm{H}, \mathrm{t}, \mathrm{NCH}_{2} \underline{\mathrm{C}}_{2} \mathrm{NEt}_{2}, J 5.9 \mathrm{~Hz}\right), 2.46\left(4 \mathrm{H}, \mathrm{q}, \mathrm{NC}_{2} \mathrm{CH}_{3}, J 7.1\right), 0.85(6 \mathrm{H}, \mathrm{t}$,

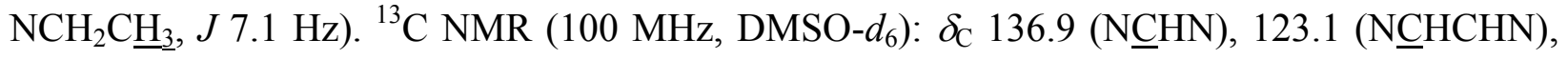
$122.6(\mathrm{NCH} \underline{\mathrm{HN}}), 51.8\left(\mathrm{NCH}_{2} \underline{\mathrm{CH}_{2}} \mathrm{NEt}_{2}\right), 47.3\left(\mathrm{NCH}_{2} \mathrm{CH}_{3}\right), 46.2\left(\mathrm{NCH}_{2} \mathrm{CH}_{3}\right), 35.6\left(\mathrm{NCH}_{3}\right)$, $11.6\left(\mathrm{NCH}_{2} \mathrm{CH}_{3}\right)$. ESI-HRMS: $m / z[\mathrm{M}]^{+}$calcd for $\mathrm{C}_{10} \mathrm{H}_{20} \mathrm{~N}_{3}{ }^{+}: 182.1657$. Found: 182.1653 .

1,3-Dimethylimidazolium dimethyl phosphate (3) was synthesized in a similar manner as reported previously by our group. ${ }^{20}$ FTIR (ATR film), $v_{\max }\left(\mathrm{cm}^{-1}\right): 1577(\mathrm{C}=\mathrm{C}), 1242(\mathrm{P}=\mathrm{O})$, $1040(\mathrm{P}-\mathrm{O}) .{ }^{1} \mathrm{H}$ NMR $\left(400 \mathrm{MHz}, \mathrm{DMSO}-d_{6}\right): \delta_{\mathrm{H}} 9.38(1 \mathrm{H}, \mathrm{s}, \mathrm{NC} \underline{\mathrm{HN}}), 7.73(2 \mathrm{H}, \mathrm{d}, \mathrm{NC} \underline{\mathrm{HC}} \underline{\mathrm{HN}}, J$ $1.5 \mathrm{~Hz}), 3.85\left(6 \mathrm{H}, \mathrm{s}, \mathrm{NC}_{3}\right), 3.27\left(6 \mathrm{H}, \mathrm{d}, \mathrm{OCH}_{3}, J 10.3 \mathrm{~Hz}\right) .{ }^{13} \mathrm{C} \mathrm{NMR}\left(100 \mathrm{MHz}, \mathrm{DMSO}-d_{6}\right): \delta_{\mathrm{C}}$ $137.6(\mathrm{~N} \underline{\mathrm{CHN}}), 123.4(\mathrm{~N} \underline{\mathrm{CH}} \underline{\mathrm{CHN}}), 51.3\left(\mathrm{O}_{\mathrm{CH}}\right), 51.2\left(\mathrm{O}_{3} \mathrm{H}_{3}\right), 35.6\left(\mathrm{NC}_{3}\right)$. ESI-HRMS: $m / z$ $[\mathrm{M}]^{+}$calcd for $\mathrm{C}_{5} \mathrm{H}_{9} \mathrm{~N}_{2}{ }^{+}$: 97.0766. Found: 97.0763.

1,4-Bis-(3-methylimidazolium-1-yl)butane bis(dimethyl phosphate) (4) was synthesized in a similar manner as reported previously by our group. ${ }^{20}$ FTIR (ATR film), $v_{\max }\left(\mathrm{cm}^{-1}\right): 1579$ $(\mathrm{C}=\mathrm{C}), 1241(\mathrm{P}=\mathrm{O}), 1037(\mathrm{P}-\mathrm{O}) .{ }^{1} \mathrm{H}$ NMR (400 MHz, DMSO-d $): \delta_{\mathrm{H}} 9.47(2 \mathrm{H}, \mathrm{s}, \mathrm{NC} \underline{\mathrm{HN}}), 7.82$ $(2 \mathrm{H}, \mathrm{s}, \mathrm{NC} \underline{\mathrm{HCHN}}), 7.72(2 \mathrm{H}, \mathrm{s}, \mathrm{NCHC} \underline{\mathrm{HN}}), 4.24\left(4 \mathrm{H}, \mathrm{s}, \mathrm{NC}_{\underline{H}}\left(\mathrm{CH}_{2}\right)_{2} \underline{\mathrm{C}}_{2} \mathrm{~N}\right), 3.86(6 \mathrm{H}, \mathrm{s}$, $\left.\mathrm{NC}_{3}{ }_{3}\right), 3.28\left(12 \mathrm{H}, \mathrm{d}, \mathrm{OC}_{3}, J 10.3 \mathrm{~Hz}\right), 1.79\left(4 \mathrm{H}, \mathrm{s}, \mathrm{NCH}_{2}\left(\mathrm{C}_{2} \underline{2}_{2}{ }_{2} \mathrm{CH}_{2} \mathrm{~N}\right) .{ }^{13} \mathrm{C} \mathrm{NMR}(100 \mathrm{MHz}\right.$, DMSO- $\left.d_{6}\right): \delta_{\mathrm{C}} 137.2(\mathrm{NC} \underline{H N}), 123.6(\mathrm{~N} \underline{\mathrm{CHCHN}}), 122.3(\mathrm{NCH} \underline{\mathrm{HN}}), 51.3\left(\mathrm{O}^{-} \mathrm{H}_{3}\right), 47.7$ $\left(\mathrm{NCH}_{2}\left(\mathrm{CH}_{2}\right)_{2} \underline{\mathrm{CH}}_{2} \mathrm{~N}\right), 35.7\left(\mathrm{NCH}_{3}\right), 25.9\left(\mathrm{NCH}_{2}\left(\underline{\mathrm{CH}}_{2}\right)_{2} \mathrm{CH}_{2} \mathrm{~N}\right)$. ESI-HRMS: $\mathrm{m} / z[\mathrm{M}]^{+}$calcd for $\mathrm{C}_{12} \mathrm{H}_{19} \mathrm{~N}_{4}^{+}: 219.1604$. Found: 219.1603 .

1-[2-(Diethylmethylammonium)ethyl]-3-methylimidazolium bis(dimethyl phosphate) (5). A solution of 1-[2-(diethylamino)ethyl]-3-methylimidazolium chloride (2) (3.89 g, $17.9 \mathrm{mmol})$ and TMP (7.51 g, $53.6 \mathrm{mmol})$ in $\mathrm{MeCN}(7 \mathrm{~mL})$ was stirred at $80^{\circ} \mathrm{C}$ for $72 \mathrm{~h}$. After the completion of the reaction, solvent was removed by rotary evaporation $\left(10 \mathrm{mbar}, 70{ }^{\circ} \mathrm{C}\right)$. Diethyl ether $(5 \mathrm{~mL})$ was added to the crude product and the mixture was stirred for $5 \mathrm{~min}$. The diethyl ether layer was decanted and the procedure was repeated using toluene $(7 \times 5 \mathrm{~mL})$ and heating the mixture to reflux. The toluene layer was decanted while hot. Any remaining solvent was then removed by 
rotary evaporation $\left(10 \mathrm{mbar}, 70{ }^{\circ} \mathrm{C}\right)$. The pure product was dried under vacuum $(0.6 \mathrm{mbar}, 80$ ${ }^{\circ} \mathrm{C}, 12 \mathrm{~h}$ ) and was subjected to $\mathrm{AgNO}_{3}$ test to confirm the absence of starting material.

IL 5. White solid, yield $99 \%, 7.94 \mathrm{~g}, \mathrm{mp} 79{ }^{\circ} \mathrm{C}$ (determined via DTA/TG); FTIR (ATR film), $v_{\max }\left(\mathrm{cm}^{-1}\right): 1579(\mathrm{C}=\mathrm{C}), 1240(\mathrm{P}=\mathrm{O}), 1036(\mathrm{P}-\mathrm{O}) .{ }^{1} \mathrm{H}$ NMR $\left(400 \mathrm{MHz}, \mathrm{CDCl}_{3}\right): \delta_{\mathrm{H}} 10.67(1 \mathrm{H}, \mathrm{s}$, $\mathrm{NC} \underline{\mathrm{HN}}), \quad 8.86(1 \mathrm{H}, \quad \mathrm{s}, \quad \mathrm{NC} \underline{\mathrm{HCHN}}), \quad 7.15(1 \mathrm{H}, \quad \mathrm{s}, \quad \mathrm{NCHC} \underline{\mathrm{HN}}), \quad 5.25-5.16 \quad(2 \mathrm{H}, \mathrm{m}$, $\left.\mathrm{NC}_{2} \mathrm{CH}_{2} \mathrm{NCH}_{3} \mathrm{Et}_{2}\right), 4.28-4.19\left(2 \mathrm{H}, \mathrm{m}, \mathrm{NCH}_{2} \underline{\mathrm{CH}}_{2} \mathrm{NCH}_{3} \mathrm{Et}_{2}\right), 3.91\left(3 \mathrm{H}, \mathrm{s}, \mathrm{NC}_{3}\right), 3.79-3.68(2 \mathrm{H}$, $\left.\mathrm{m}, \mathrm{NC}_{2} \mathrm{CH}_{3}\right), 3.67-3.58\left(2 \mathrm{H}, \mathrm{m}, \mathrm{NC}_{2} \mathrm{CH}_{3}\right), 3.55\left(12 \mathrm{H}, \mathrm{d}, \mathrm{OCH}_{3}, J 10.5 \mathrm{~Hz}\right), 3.27(3 \mathrm{H}, \mathrm{s}$, $\left.\mathrm{NCH}_{2} \mathrm{CH}_{2} \mathrm{NCH}_{3} \mathrm{Et}_{2}\right), 1.36\left(6 \mathrm{H}, \mathrm{t}, \mathrm{NCH}_{2} \underline{\mathrm{CH}}_{3}, J 7.2 \mathrm{~Hz}\right) .{ }^{13} \mathrm{C} \mathrm{NMR}\left(100 \mathrm{MHz}, \mathrm{CDCl}_{3}\right): \delta_{\mathrm{C}} 139.7$

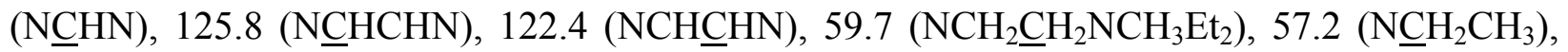
$52.7\left(\mathrm{OCH}_{3}\right), 52.6\left(\mathrm{OCH}_{3}\right), 47.4\left(\mathrm{NCH}_{2} \mathrm{CH}_{2} \mathrm{NCH}_{3} \mathrm{Et}_{2}\right), 42.5\left(\mathrm{NCH}_{2} \mathrm{CH}_{2} \mathrm{NCH}_{3} \mathrm{Et}_{2}\right), 36.4\left(\mathrm{NCH}_{3}\right)$, $8.2\left(\mathrm{NCH}_{2} \mathrm{CH}_{3}\right)$. ESI-HRMS: $m / z[\mathrm{M}]^{+}$calcd for $\mathrm{C}_{11} \mathrm{H}_{22} \mathrm{~N}_{3}{ }^{+}:$196.1814. Found: 196.1790 .

1-[2-(Diethylamino)ethyl]-3-methylimidazolium chloride hydrochloride (9). 1-Methylimidazole (3.98 g, $48.5 \mathrm{mmol})$ and 2-(diethylamino)ethyl chloride hydrochloride (8.35 g, 48.5 mmol) were dissolved in $\mathrm{MeCN}(30 \mathrm{~mL}$ ) and refluxed for $48 \mathrm{~h}$. After cooling to rt, the colorless precipitate was filtered, washed on the filter with EtOAc $(15 \mathrm{~mL})$, and recrystallized from EtOH. The pure product was dried under vacuum $\left(0.6 \mathrm{mbar}, 100^{\circ} \mathrm{C}, 5 \mathrm{~h}\right)$.

IL 9. Colorless solid, yield $66 \%, 8.09 \mathrm{~g}, \mathrm{mp} 197-200{ }^{\circ} \mathrm{C}$ (from EtOH); FTIR (ATR film), $v_{\max }$ $\left(\mathrm{cm}^{-1}\right): 3449(\mathrm{NH}), 1641(\mathrm{C}=\mathrm{N}), 1576(\mathrm{C}=\mathrm{C}) .{ }^{1} \mathrm{H}$ NMR $\left(400 \mathrm{MHz}, \mathrm{DMSO}-d_{6}\right): \delta_{\mathrm{H}} 11.40(1 \mathrm{H}, \mathrm{s}$, $\mathrm{N} \underline{\mathrm{H}}), 9.45(1 \mathrm{H}, \mathrm{s}, \mathrm{NC} \underline{\mathrm{HN}}), 7.99(1 \mathrm{H}, \mathrm{s}, \mathrm{NC} \underline{H C H N}), 7.75(1 \mathrm{H}, \mathrm{s}, \mathrm{NCHC} \underline{\mathrm{HN}}), 4.73(2 \mathrm{H}, \mathrm{t}$, $\left.\mathrm{NC}_{2} \mathrm{CH}_{2} \mathrm{NHEt}_{2}, J 6.6 \mathrm{~Hz}\right), 3.85\left(3 \mathrm{H}, \mathrm{s}, \mathrm{NC}_{3}\right), 3.60\left(2 \mathrm{H}, \mathrm{d}, \mathrm{NCH}_{2} \underline{\mathrm{C}}_{2} \mathrm{NHEt}_{2}, J 4.9 \mathrm{~Hz},\right), 3.16$ $\left(4 \mathrm{H}, \mathrm{s}, \mathrm{NC}_{2} \mathrm{CH}_{3}\right), 1.24\left(6 \mathrm{H}, \mathrm{t}, \mathrm{NCH}_{2} \mathrm{C}_{3}, J 7.2 \mathrm{~Hz}\right) .{ }^{13} \mathrm{C} \mathrm{NMR}\left(100 \mathrm{MHz}, \mathrm{DMSO}-d_{6}\right): \delta_{\mathrm{C}} 137.8$

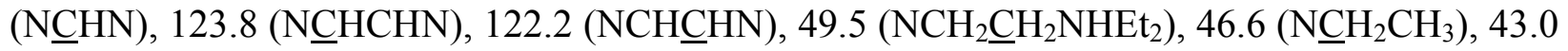
$\left.\left(\mathrm{N}^{\mathrm{C}} \mathrm{H}_{2} \mathrm{CH}_{2} \mathrm{NHEt}_{2}\right), 35.9\left(\mathrm{NCH}_{3}\right), 8.2\left(\mathrm{NCH}_{2} \underline{\mathrm{CH}}\right)_{3}\right)$. ESI-HRMS: $\mathrm{m} / z[\mathrm{M}]^{+}$calcd for $\mathrm{C}_{10} \mathrm{H}_{20} \mathrm{~N}_{3}{ }^{+}$: 182.1657. Found: 182.1651.

General procedure for the Knoevenagel condensation in ionic liquids 1-5. 4(Dimethylamino)benzaldehyde (6) $(120 \mathrm{mg}, 0.80 \mathrm{mmol})$ was dissolved in the appropriate IL (8.0 mmol) at $80{ }^{\circ} \mathrm{C}$ temperature and ethyl cyanoacetate (7) $(91 \mathrm{mg}, 0.80 \mathrm{mmol})$ was added under vigorous stirring. After specified time (Figure 2), 1-2 drops of the reaction mixture were quenched with EtOAc/brine $(1 \mathrm{~mL} / 0.3 \mathrm{~mL})$ mixture at $-10{ }^{\circ} \mathrm{C}$. The EtOAc layer was separated and the brine layer was repeatedly extracted with EtOAc $(9 \times 1 \mathrm{~mL})$ at $-10{ }^{\circ} \mathrm{C}$. The combined extracts were concentrated to approximately $1 \mathrm{~mL}$ by rotary evaporation $\left(10 \mathrm{mbar}, 30^{\circ} \mathrm{C}\right)$ and the conversion of the aldehyde $\mathbf{6}$ was calculated from the data obtained via gas chromatography. To support the results obtained by gas chromatography, the reaction was performed in ILs 2-5 according to the general procedure and product 8 was isolated. After stirring at $80{ }^{\circ} \mathrm{C}$ for $40 \mathrm{~min}$, cold distilled water $(10 \mathrm{~mL})$ was added to the reaction mixture. The crude product was filtered, washed on the filter with distilled water $(10 \mathrm{~mL})$ and recrystallized from $\mathrm{EtOH} / \mathrm{H}_{2} \mathrm{O}$. The pure product was dried under vacuum $\left(0.6 \mathrm{mbar}, 65^{\circ} \mathrm{C}, 2 \mathrm{~h}\right)$. The isolated yields of 8 were in a full agreement with the results provided by gas chromatography. 
In IL 2: yield $160 \mathrm{mg}(80 \%)$; in IL 3: yield $186 \mathrm{mg}$ (93\%), in IL 4, yield $174 \mathrm{mg}$ (87\%); in IL 5, yield $188 \mathrm{mg}$ (94\%). Yellow solid, mp $125-126{ }^{\circ} \mathrm{C}$ (from $\mathrm{EtOH} / \mathrm{H}_{2} \mathrm{O}$ ); FTIR (ATR film), $v_{\max }$ $\left(\mathrm{cm}^{-1}\right): 2208(\mathrm{C} \equiv \mathrm{N}), 1701(\mathrm{C}=\mathrm{O}), 1610(\mathrm{C}=\mathrm{C}) .{ }^{1} \mathrm{H} \mathrm{NMR}\left(400 \mathrm{MHz}, \mathrm{CDCl}_{3}\right): \delta_{\mathrm{H}} 8.07(1 \mathrm{H}, \mathrm{s}$, $\mathrm{C} \underline{H}=\mathrm{C}), 7.96-7.90\left(2 \mathrm{H}, \mathrm{m}, \underline{\mathrm{H}}_{\mathrm{ar}}\right), 6.72-6.66\left(2 \mathrm{H}, \mathrm{m}, \underline{\mathrm{H}}_{\mathrm{ar}}\right), 4.33\left(2 \mathrm{H}, \mathrm{q}, \mathrm{OC}_{2} \mathrm{CH}_{3}, J 7.1 \mathrm{~Hz}\right), 3.10$ $\left(6 \mathrm{H}, \mathrm{s}, \mathrm{N}\left(\mathrm{C}_{3}{ }_{2} 2\right), 1.37\left(3 \mathrm{H}, \mathrm{t}, \mathrm{OCH}_{2} \underline{\mathrm{C}}_{3}, J 7.1 \mathrm{~Hz}\right) .{ }^{13} \mathrm{C} \mathrm{NMR}\left(100 \mathrm{MHz}, \mathrm{CDCl}_{3}\right): \delta_{\mathrm{C}} 164.4\right.$ $(\underline{\mathrm{C}}=\mathrm{O}), 154.6(\underline{\mathrm{CH}}=\mathrm{C}), 153.7\left(\mathrm{NC}_{\mathrm{Car}}\right), 134.2\left(\underline{\mathrm{C}}_{\mathrm{ar}}\right), 119.6\left(\underline{\mathrm{C}}_{\mathrm{ar}} \mathrm{CH}=\mathrm{C}\right), 117.7(\underline{\mathrm{CN}}), 111.7\left(\underline{\mathrm{C}}_{\mathrm{ar}}\right), 94.3$ $(\mathrm{CH}=\underline{\mathrm{C}}), 62.0\left(\mathrm{OCH}_{2} \mathrm{CH}_{3}\right), 40.2\left(\mathrm{~N}\left(\underline{\mathrm{CH}}_{3}\right)_{2}\right), 14.4\left(\mathrm{OCH}_{2} \underline{\mathrm{CH}}_{3}\right)$. ESI-HRMS: $m / z[\mathrm{M}+\mathrm{H}]^{+}$calcd for $\mathrm{C}_{14} \mathrm{H}_{17} \mathrm{~N}_{2} \mathrm{O}_{2}^{+}: 245.1285$. Found: 245.1286 .

\section{Supplementary Material}

${ }^{1} \mathrm{H}$ and ${ }^{13} \mathrm{C}$ NMR spectra of ILs 1-5, salt 9, and compound $\mathbf{8}$.

\section{Acknowledgements}

This research was supported by the European Social Foundation (ESF), project Nr.1DP/1.1.1.2.0/13/APIA/VIAA/011. Authors gratefully acknowledge Dr. Ilva Nakurte and Ms. Diana Dzabijeva (University of Latvia) for HRMS analyses, Mr. Toms Rekis (University of Latvia) for DTA/TG analyses, Dr. Juris Popelis (Latvian Institute of Organic Synthesis) for NMR analyses and Mr. Eduards Bakis (Imperial College London) for the valuable discussions.

\section{References}

1. Chen, X.; Ying, A. In Ionic Liquids: Applications and Perspectives; Kokorin, A. Ed.; InTech: Rijeka, 2011; pp 305-330.

2. McCluskey, A.; Robinson, P. J.; Hill, T.; Scott, J. L.; Edwards, J. K. Tetrahedron Lett. 2002, $43,3117$. http://dx.doi.org/10.1016/S0040-4039(02)00480-X

3. Venkat Narsaiah, A.; Basak, A. K.; Visali, B.; Nagaiah, K. Synth. Commun. 2004, 34, 2893. http://dx.doi.org/10.1081/SCC-200026625

4. Han, J.; Xu, Y.; Su, Y.; She, X.; Pan, X. Catal. Commun. 2008, 9, 2077. http://dx.doi.org/10.1016/j.catcom.2008.04.006

5. Saravanamurugan, S.; Palanichamy, M.; Hartmann, M.; Murugesan, V. Appl. Catal. A: General 2006, 298, 8.

http://dx.doi.org/10.1016/j.apcata.2005.09.014

6. Bartoli, G.; Bosco, M.; Carlone, A.; Dalpozzo, R.; Galzerano, P.; Melchiorre, P.; Sambri, L. Tetrahedron Lett. 2008, 49, 2555. 
http://dx.doi.org/10.1016/j.tetlet.2008.02.093

7. Ajani, O. O.; Nwinyi, O. C. J. Heterocycl. Chem. 2010, 47, 179.

8. De-la-Torre, P.; Osorio, E.; Alzate-Morales, J. H.; Caballero, J.; Trilleras, J.; AstudilloSaavedra, L.; Brito, I.; Cárdenas, A.; Quiroga, J.; Gutiérrez, M. Ultrason. Sonochem. 2014, $21,1666$. http://dx.doi.org/10.1016/j.ultsonch.2014.02.021

9. Anastas, P. T.; Warner, J. C. Green Chemistry: Theory and Practice; Oxford University Press: Oxford, 2000; pp 1-152.

10. Hallett, J. P.; Welton, T. Chem. Rev. 2011, 111, 3508. http://dx.doi.org/10.1021/cr1003248

11. Plechkova, N. V.; Seddon, K. R. Chem. Soc. Rev. 2008, 37, 123. http://dx.doi.org/10.1039/B006677J

12. Menegatti, R. In Green Chemistry - Environmentally Benign Approaches; Kidwai, M.; Mishra, N. K. Eds.; InTech: Rijeka, 2012; pp 13-32. http://dx.doi.org/10.5772/1996

13. Hu, X.-M.; Zhao, Y.; Gao, Y.-F.; Xiao, Y.-B.; Zhang, B.-X. Adv. Mat. Res. 2012, 554-556, 557.

http://dx.doi.org/10.4028/www.scientific.net/AMR.554-556.557

14. Anderson, J. L.; Ding, R.; Ellern, A.; Armstrong, D. W. J. Am. Chem. Soc. 2005, 127, 593. http://dx.doi.org/10.1021/ja046521u

15. Shirota, H.; Mandai, T.; Fukazawa, H.; Kato, T. J. Chem. Eng. Data 2011, 56, 2453. http://dx.doi.org/10.1021/je2000183

16. Steudte, S.; Bemowsky, S.; Mahrova, M.; Bottin-Weber, U.; Tojo-Suarez, E.; Stepnowski, P.; Stolte, S. RSC Adv. 2014, 4, 5198. http://dx.doi.org/10.1039/c3ra45675g

17. Jawale, D. V.; Pratap, U. R.; Lingampalle, D. L.; Mane, R. A. Chin. J. Chem. 2011, $29,942$. http://dx.doi.org/10.1002/cjoc.201190192

18. Luo, J.; Xin, T.; Wang, Y. New J. Chem. 2013, 37, 269. http://dx.doi.org/10.1039/C2NJ40890B

19. Godajdar, B. M.; Kiasat, A. R.; Hashemi, M. M. Heterocycles 2013, 87, 559. http://dx.doi.org/10.3987/COM-12-12626

20. Priede, E.; Bakis, E.; Zicmanis, A. Synlett 2014, 25, 2447. http://dx.doi.org/10.1055/s-0034-1379018

21. Zhang, S.; Goncalves, L. D.; Lefebvre, H.; Tessier, M.; Rousseau, B.; Fradet, A. ACS Macro Lett. 2012, 1, 1079. http://dx.doi.org/10.1021/mz300264v

22. Zicmanis, A.; Anteina, L. Tetrahedron Lett. 2014, 55, 2027. http://dx.doi.org/10.1016/j.tetlet.2014.02.035

23. Hammett, L. P. J. Am. Chem. Soc. 1937, 59, 96. http://dx.doi.org/10.1021/ja01280a022 
24. Zhang, L.; Yang, Y.; Xue, Y.; Fu, X.; An, Y; Gao, G. Catal. Today 2010, 158, 279. http://dx.doi.org/10.1016/j.cattod.2010.03.060

25. Forsyth, S. A.; Fröhlich, U.; Goodrich, P.; Nimal Gunaratne, H. Q.; Hardacre, C.; McKeown, A.; Seddon, K. R. New J. Chem. 2010, 34, 723. http://dx.doi.org/10.1039/b9nj00729f

26. Sowmiah, S.; Srinivasadesikan, V.; Tseng, M.-C.; Chu, Y.-H. Molecules 2009, 14, 3780. http://dx.doi.org/10.3390/molecules14093780

27. Aggarwal, V. K.; Emme, I.; Mereu, A. Chem. Commun. 2002, 1612. http://dx.doi.org/10.1039/b203079a

28. Chen, X.; Song, H.; Chen, P.; Wang, F.; Qian, Y.; Li, X. Acta Chim. Sinica 2012, 70, 770. http://dx.doi.org/10.6023/A1108223

29. Armarego, W. L. F.; Chai, C. L. L. Purification of Laboratory Chemicals, 5th Edn.; Elsevier Science: Bodmin, 2003; pp 80-388.

30. Ab Rani, M. A.; Brant, A.; Crowhurst, L.; Dolan, A.; Lui, M.; Hassan, N. H.; Hallett, J. P.; Hunt, P. A.; Niedermeyer, H.; Perez-Arlandis, J. M.; Schrems, M.; Welton, T.; Wilding, R. Phys. Chem. Chem. Phys. 2011, 13, 16831.

http://dx.doi.org/10.1039/c1cp21262a 